\title{
A comparison of the prognostic significance of changes in NT-proBNP levels in HFrEF and HFpEF.
}

\author{
Nishant Sahni ${ }^{1}$, Umesh Sharma ${ }^{1}$, and Rashi Arora ${ }^{1}$ \\ ${ }^{1}$ Mayo Clinic Arizona
}

April 30, 2021

\begin{abstract}
Background: Rising NT-proBNP are associated with reduced survival patients with HFrEF. However, it remains to be conclusively and formally demonstrated that the temporal trend in NT-proBNP level carries prognostic significance in HFpEF. Objective: To determine whether there is an association between rising NT-proBNP levels and 6-month survival in patients with HFpEF and HFrEF. Methods: We examined a cohort of 5203 patients to 5 hospitals in a regional health care system who had at least one admission to the hospital with diagnoses of heart failure over a 3-year period. Kaplan-Meier survival curves were constructed for patients with downtrending ( $>25 \%$ net decrease), stable or uptrending ( $>25 \%$ net increase) NT-proBNP levels in HF, HFpEF and HFrEF patients. The log-rank test was used to test for differences in 6-month survival amongst the groups. Multivariate extended Cox regression models were constructed for 6-month survival with NT-proBNP as a time-varying covariate. Age, albumin, sex, race, serum creatinine, systolic and diastolic blood pressures and Charlson comorbidity scores at baseline were used as covariates in the model. Separate analyses were done for HFpEF and HFrEF patients. Results: HFpEF and HFrEF patients with up-trending levels had significantly lower 6-month survival rates than patients with downtrending or stable NT-proBNP levels. A doubling of the NT-proBNP level in patients was significantly associated with reduced 6-month survival in patients with in both subgroups of HF, HFpEF and HFrEF (HFpEF-HR: 1.53(1.49-2.57), HFrEF HR: 1.45(1.43-1.48) after adjusting for covariates.
\end{abstract}

A comparison of the prognostic significance of changes in NT-proBNP levels in HFrEF and HFpEF.

Nishant Sahni MD, MS, Umesh Sharma MD, Rashi Arora MD

*Division of Hospital Medicine, Mayo Clinic , Arizona

Corresponding Author: Nishant Sahni MD,MS, email:

sahni.nishant@mayo.edu

Conflict of interest statement: None of the authors have any conflicts of interest to disclose.

Abbreviations:

CHF: Congestive Heart Failure

EMR: Electronic Medical Record

HF: Heart Failure

HFpEF: Heart Failure with preserved Ejection Fraction

HFrEF: Heart Failure with reduced Ejection Fraction

HR: Hazard Ratio 
NP: Natriuetic Peptide

NT-proBNP: N terminal pro-Brain Natriuretic Peptide

Echo: Echocardiogram

sd: standard deviation

ICD9: International Classification of Diseases

IQR: Interquartile Range

\section{Abstract.}

\section{Background:}

Rising NT-proBNP are associated with reduced survival patients with HFrEF. However, it remains to be conclusively and formally demonstrated that the temporal trend in NT-proBNP level carries prognostic significance in HFpEF.

Objective: To determine whether there is an association between rising NT-proBNP levels and 6-month survival in patients with HFpEF and HFrEF.

\section{Methods:}

We examined a cohort of 5203 patients to 5 hospitals in a regional health care system - who had at least one admission to the hospital with diagnoses of heart failure over a 3-year period. Kaplan-Meier survival curves were constructed for patients with downtrending ( $>25 \%$ net decrease), stable or uptrending ( $>25 \%$ net increase) NT-proBNP levels in HF, HFpEF and HFrEF patients. The log-rank test was used to test for differences in 6-month survival amongst the groups. Multivariate extended Cox regression models were constructed for 6-month survival with NT-proBNP as a time-varying covariate. Age, albumin, sex, race, serum creatinine, systolic and diastolic blood pressures and Charlson comorbidity scores at baseline were used as covariates in the model. Separate analyses were done for HFpEF and HFrEF patients.

\section{Results:}

HFpEF and HFrEF patients with up-trending levels had significantly lower 6-month survival rates than patients with downtrending or stable NT-proBNP levels. A doubling of the NT-proBNP level in patients was significantly associated with reduced 6-month survival in patients with in both subgroups of HF, HFpEF and HFrEF (HFpEF-HR: 1.53(1.49-2.57), HFrEF HR: 1.45(1.43-1.48) after adjusting for covariates.

\section{What's known about the topic ?}

-A rise in NT-proBNP levels is accompanied by clinical deterioration in patients who have heart failure with with a reduced ejection fraction. It is has been previously been unclear to what extent this hold true for patients who have heart failure with a preserved ejection fraction.

\section{What our study adds?}

-We show that similar to HF with a reduced ejection fraction - up-trending NT-proBNP levels accompany clinical deterioration in patients with HF with preserved ejection fraction. The risk of clinical deterioration with rising NT-proBNP levels is of a similar magnitude in both subsets of heart failure.

\section{Introduction.}

Around 5.1 million patients in the US have HF, the estimated cost of HF to the economy is estimated to be around $\$ 30.7$ billion dollars a year.1 Even though the past few decades have seen significant advances in the understanding of the HF syndrome, patients with chronic HF have still an adverse prognosis overtime. In the last decade serum NT-proBNP has emerged as a widely used biomarker in HF. The N-terminal prohormone of brain natriuretic peptide (NT-proBNP) is a prohormone with a 76 amino acid N-terminal inactive protein 
that is cleaved from the molecule to release brain natriuretic peptide. ${ }^{2,3}$ It is secreted by cardiac myocytes in response to bio-mechanical stress.

Numerous studies have evaluated "point in time" measurements of NT-proBNP. Higher NT-proBNP levels are clearly associated with a prognosis in acute and chronic HF (both HFPeF and HFReF). ${ }^{1}$ There has been considerable enthusiasm and controversy around using NTpro-BNP levels to guide the management of $\mathrm{HF}^{1,2}$. This biomarker strategy relies on a better understanding of the association of temporal trends in NTpro-BNP levels in patients and clinical outcomes ${ }^{1,2}$. Serial assessment of NP in HF has revealed that changes in NP concentrations over time parallels clinical outcomes both in patients with chronic HF and acute decompensated heart failure ${ }^{3,4,5}$. However, it is unclear whether this holds true in patients with both subsets of HF- $\mathrm{HpEF}$ (left ventricular ejection fraction (LVEF) > 50\%) and HFrEF (left ventricular ejection fraction $(\mathrm{LVEF})<40 \%)^{6}$.

Some investigators have suggested that the association of temporal trends in NT-proBNP levels and clinical outcomes (that is observed in HFrEF) may not be observed in patients with HFpEF, partly because there may be significant difference in the kinetics of NT-proBNP in HFpEF and HFrEF. ${ }^{6,7}$ NT-proBNP levels in patients with HFpEF have been shown to have a significantly higher short-term "intra-day" fluctuation than in patients HFrEF, without any discernible change in clinical status. ${ }^{7}$ This suggests that longer term trends in NT-proBNP levels in HFpEF patients, may not be as tightly associated with clinical outcomes as in $\mathrm{HFrEF}$.

We conducted this retrospective study to investigate whether temporal trends in NT-pro-BNP levels were associated with mortality in both HFpEF and/or HFrEF. We had the following hypothesis; Rising NTproBNP levels would be associated with a lower six-month survival in both the echocardiographically defined subsets of HF (HFpEF and HFrEF) after adjusting for other clinically relevant co-variates.

Figure 1) Study structure and patient inclusion criterion.

\section{Methods}

After obtaining approval from our institutional review board we used data from our institutional clinical data warehouse to create a dataset of 5203 patients who had at-least one admission to the hospital or were treated in the ER for a primary diagnosis of CHF (ICD9 diagnosis of 402.01, 402.11, 402.91, 404.01, 404.11, 404.91, 428.0, 428.1, 428.20, 428.21, 428.22, 428.23, 428.30, 428.31, 428.32, 428.33, 428.40, 428.41, 428.42, 428.43 , or 428.9 tied to an encounter with an encounter date of $1 / 1 / 2011$ or later in which the patient was $>=18$ years old at the time of the encounter and in which the ICD9 diagnosis code is a primary diagnosis) at one of the 5 hospitals in our hospital system over a period of 3 years. All patients in the dataset had at least one Transthoracic Echocardiogram (TTE) with an assessment of the ejection fraction (EF) along with at least one NT-proBNP measurement throughout this period (either as an inpatient or outpatient). Patients with end stage renal disease on renal replacement therapy $(585.5,585.6,403.11,403.91,404.02,404.03$, 404.12,404.13,404.92,404.93,V45.11, V45.12, V42.0) and patients who had follow-up for less than 6 months were excluded from the dataset.(Figure 1) The patient's date of entry into the study was taken as the date of the first available NT-proBNP measurement during the 3-year study period. Patients received NT-pro-BNP levels at variable periods and frequencies across the study period according to the discretion of the treating physicians. The median interval between two successive values NT-proBNP measurements was of 14 (IQR 3-44days).

Using text-mining techniques we extracted time-stamped ejection fraction (EF) values from all the available echocardiogram reports in the EMR and verified the extraction by manual review. The final dataset contained time stamped NT-proBNP measurements along with the nearest available albumin, creatinine, systolic BP (SBP), diastolic BP(DBP) measurements. If there were multiple values for these data points on a particular calendar day, then the maximum value for the day was used for the patient. If the EF was reported as a range in the Echo report, then the lower end of the range was used (for e.g. if the EF was reported as 45-50\%, $45 \%$ was used). EF was measured by the biplane method (the method was specified only in $26.7 \%$ of the reports). A patient was classified has having HFpEF if their minimum EF across all their echocardiograms 
was greater than or equal to $50 \%$ and $\mathrm{HFrEF}$ if their maximum EF across all echocardiograms was below $40 \%{ }^{6}$. Patients whose EF fluctuated and who moved between the two categories were not classified within either category; instead they were classified as a separate mixed category. Patients who had more than a $25 \%$ net increase or decrease in serum NT-proBNP from their initial NT-proBNP in the study period were classified as having an up-trending or downtrending NT-proBNP levels respectively. Patients who had less than a $25 \%$ change in their pro-BNP levels were noted to have stable NT-pro-BNP values. To calculate the clinical Charlson comorbidity scores for the patients, all available ICD-CM codes, across all encounters during the study period were used. The Deyo-Quan modification of the Charlson index for was used for the calculation of the co-morbidity scores and classifying co-morbidities. ${ }^{7}$ All baseline labs, demographic and clinical variables for each patient were used from the first available measurement during the study period (baseline variables). We had multiple NT-proBNP measurements for each patient and these were treated as a time dependent co-variate in the regression. NTproBNP assays were performed using the proBNP reagent pack (Roche Diagnostics, Meylan, France) with an Elecsys immunoanalyzer (Roche).

\title{
Statistical Methods
}

The tables reported mean with standard deviation for variables that are normally distributed and median with interquartile range is reported for variables that do not have a normal distribution. For groupcomparisons we used a one-way test with a regular variance assumption for continuous and the Chi-Square test for categorical variables. For continuous non-normal variables the Kruskal test was used.

\section{Results}

\section{Demographic and clinical characteristics of patients.}

The demographic, biochemical, physiological and clinical characteristics are outlined in Table 1. Patients are stratified according to the overall trend in NT-proBNP level. The demographic and clinical characteristics of the cohort stratified on the echocardiographic phenotype of HF are in Supplementary Table 1.

6 month survival curves stratified by temporal trends of the NT-proBNP levels

Kaplan Meir survival curves stratified according to their temporal trend in their NT-proBNP level through the study period. The log-rank test was used to compare the survival curves. Separate curves were created for HF,HFpEF and HFrEF.(Figure 3)

\author{
Variables \\ Number of patients \\ Black - n (\%) \\ Male - $\mathrm{n}(\%)$ \\ Peripheral Vascular Disease - $\mathrm{n}(\%)$ \\ Dementia (\%) \\ Pulmonary disease (\%) \\ Diabetes mellitus (\%) \\ Diabetes mellitus with complications (\%) \\ Cancer (\%) \\ Liver disease- $(\%)$ \\ Metastatic malignancy (\%) \\ HIV (\%) \\ Liver disease- Mild (\%) \\ CHF (\%) \\ DHF $(\%)$ \\ SHF $(\%)$ \\ mixed $(\%)$ \\ Ischemic heart disease (\%) \\ Valvular heart (\%)
}

Down-trending
979
$61(6.2)$
$469(47.9)$
$318(32.5)$
$79(8.1)$
$719(73.4)$
$205(20.9)$
$213(21.8)$
$143(14.6)$
$29(3.0)$
$67(6.8)$
$3(0.3)$
$145(14.8)$
$979(100.0)$
$461(47.1)$
$333(34.0)$
$185(18.9)$
$701(71.6)$
$595(60.8)$


Cardiomyopathy $=$ TRUE $(\%)$

$491(50.2)$

Initial NT-proBNP (median [IQR])

$4620.00[2090.00,100$

Final NT-proBNP (median [IQR])

$1630.00[627.00,3815$

Age(years,mean (sd))

$71.94(14.71)$

Serum albumin (mean (sd))

$3.77(0.51)$

Serum creatinine (median $[\mathrm{IQR}]$ )

$1.03[0.80,1.38]$

Maximum ejection fraction $(\%$, mean (sd))

$49.39(15.16)$

Minimum ejection fraction (\%, mean (sd))

$40.59(17.35)$

Charlson comorbidity score (mean (sd))

Systolic blood pressure (mmHG,mean (sd))

Diastolic blood pressure (mean (sd))

$71.87(14.57)$

Death within 6 months $=$ TRUE $(\%)$

$70(7.2)$

Initial weight (mean (sd))

Change in weight through the study (mean (sd))

$188.50(57.56)$

$-0.58(0.20)$

Number of days between the first and last NT-proBNP (mean (sd))

$67.65(57.84)$

Number of NT-proBNP measurements per patient (mean (sd))

$3.36(1.84)$

Maximum number of days between consecutive NT-proBNP measurements per patient (mean (sd))

$52.04(47.13)$

Table 1. Demographic, clinical, biochemical and echocardiographic characteristics of patients who had stable, improving or up-trending pro-BNP levels during the study period
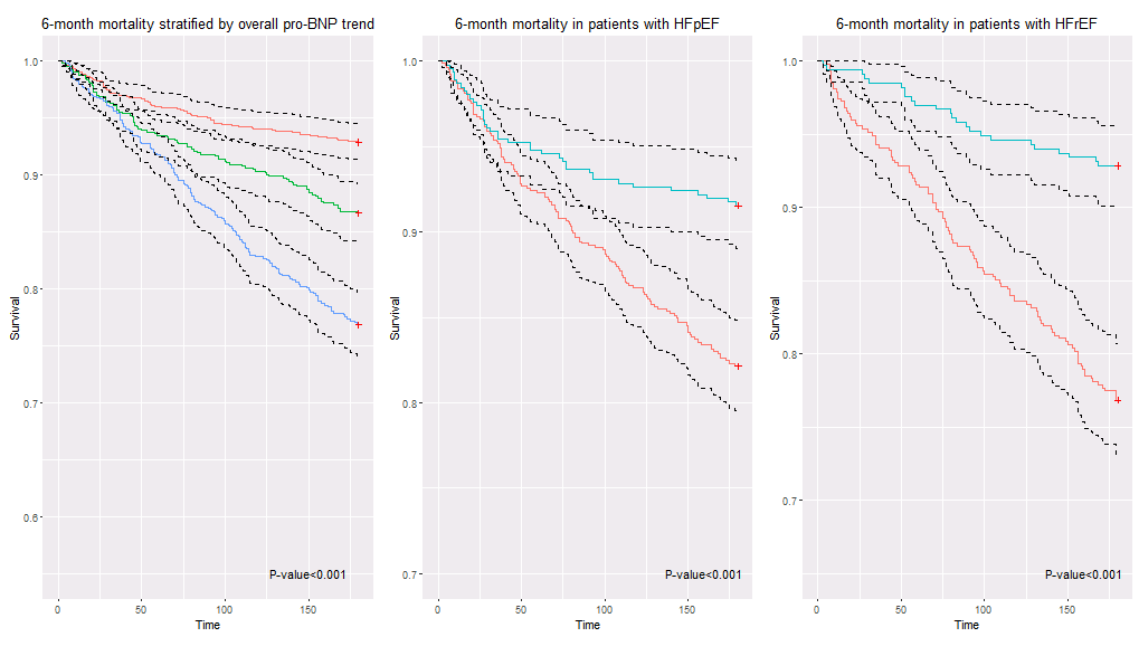

Figure 2. Kaplan-Meir estimates for survival stratified by trends in NT-proBNP. Left Panel: HF -Downtrending NT-proBNP $(>=25 \%$ decrease in NT-proBNP from initial value, red line) or stable NT$\operatorname{proBNP}$ (green, change less than $25 \%$ of initial level) and up-trending NTpro-BNP $(>=25 \%$ net rise in NT pro-BNP, blue line) .Middle panel: HFpEF - uptrending NT-proBNP (red line), downtrending or stable NT-proBNP (light blue line). Right Panel: HFrEF - uptrending NT-proBNP(red line), downtrending or stable NT-proBNP (light blue line) . p-values are reported for the logrank test.

\begin{tabular}{lllll}
\hline Term & Hazard Ratio (HFpEF) & p-value & Hazard Ratio (HFrEF) & p-value \\
Serum albumin & $0.82(0.77-0.89)$ & 0.24 & $0.96(0.91-1.01)$ & 0.74 \\
$\log 2($ NT-proBNP) & $1.53(1.49-2.57)$ & $<0.001$ & $1.45(1.43-1.48)$ & $<0.001$ \\
Age & $1.00(1.00-1.009)$ & 0.34 & $1.00(1.00-1.09)$ & 0.11 \\
Sex(Male) & $1.089(0.99-1.18)$ & 0.6 & $1.00(0.94-1.06)$ & $>0.99$ \\
Serum Creatinine & $1.19(1.12-1.26)$ & 0.013 & $1.18(1.14-1.23)$ & 0.001
\end{tabular}




\begin{tabular}{lllll} 
Black race & $1.15(0.95-1.39)$ & 0.61 & $1.11(0.97-1.27)$ & 0.62 \\
Systolic Blood Pressure & $0.99(0.99-0.996)$ & 0.044 & $0.99(0.99-1.00)$ & 0.49 \\
Diastolic Blood Pressure & $0.99(0.99-1.00)$ & 0.74 & $0.99(0.98-0.99)$ & 0.039 \\
Charlson Comorbidity scores & $1.13(1.0-1.19)$ & 0.19 & $1.00(1.00-1.08)$ & 0.47 \\
\hline
\end{tabular}

Table 2: Results of extended multivariate cox regression for six month survival with $\log 2(\mathrm{NT}$ proBNP) as a time varying co-variate.

\section{Factors associated with 6-month survival in patients with HFpEF and HFrEF.}

To analyze whether the trends in NT-proBNP level were associated with 6-month survival we used the extended Cox proportional hazards regression model. NT-proBNP was treated as a time varying co-variate. For each NT-proBNP measurement that was obtained the nearest available albumin value, creatinine, SBP and DBP values were used. Charlson Co-morbidity scores were based on all the ICD-CM codes available for all the encounters during the study period. The results of the multivariate Cox regression analysis are in Table 2. For binary and categorical variables, dummy variables were used. Observations with missing values were not used in model construction. Variables were selected for inclusion in the model based on clinical relevance in prior HF literature and consensus amongst the authors. Log(2) of the NT-proBNP was used because the distribution of NT-proBNP levels was skewed, in addition this allowed us to estimate the hazard ratio (HR) for each doubling of the NT-proBNP level (this formulation may be easier for clinicians to intuit).

6-month survival rates amongst groups of patients with improving, stable and worsening NTproBNP values. There was progressively a lower rate of 6 -month survival within the three categories of temporal trends in NT-proBNP (6-month survival in HF: down-trending (23.2\%), stable (13.4\%) and up-trending (7.2\%), Table 1).

\section{Discussion}

Unlike, the relatively slow evolving trends (over days or weeks) in plasma BNP observed in HFrEF patients, some HFpEF patients exhibited rapid spikes and falls in plasma BNP occurring at intervals of hours to one or 2 two days- without clinical acute decompensated heart failure. ${ }^{8-10}$ Some have suggested that this pattern makes NT-proBNP an unreliable marker of clinical status in HFpEF, because the levels of NT-proBNP are "spontaneously" variable in the absence of any discernible clinical change in HFpEF patients. ${ }^{8}$

We clearly demonstrate that similar to HFrEF patients, up-trending NT-proBNP levels are associated with clinical deterioration in HFpEF patients. (Figure 2). We disprove the hypothesis that rising NT-proBNP levels are somehow "decoupled" from clinical deterioration in the HFpEF due to kinetics of the molecule in this condition, as some prior studies have postulated. ${ }^{4}$ Using Cox regression that treats NT-proBNP as a time dependent covariate, we demonstrate that for both subsets of HF (HFpEF and HFrEF): a two-fold increase in the NT-proBNP levels (above normal) is associated with an increased the HR of death within 6 months by approximately $45-50 \%$ (Table 3 ).

There are several caveats to consider when interpreting the data. Our data should not be interpreted to prove that trends in NT-proBNP can be used to predict the clinical trajectory of HF. It is likely that patients with progressively rising NT-proBNP levels had certain subtypes of disease that was more likely to be refractory to treatment and thus the HF was more likely to progress. The comorbidity profiles, albumin, creatinine and the Charlson co-morbidity score were not significantly different across the groups at baseline. (Table 1) We did not match the cohorts at baseline in terms of functional status or other variables- it is likely that patients with up-trending NT-proBNP levels had a poorer functional status (NYHA class) at the time of entry into the study. Because our study was not prospective or randomized, the frequency and the period at which serial NT-proBNP measurements were obtained was not fixed and were obtained at varying periods by the patient's physicians - we used statistical methods that account for this. Although multivariable 
statistical models were used to adjust for heterogeneity between groups in this observational study, residual unmeasured confounding factors may be present.

\section{Conclusion}

Due to a high variability of NT-proBNP levels in the absence of clinical change in HFpEF patients, previous studies have raised concerns the association between temporal trends in NT-proBNP disease worsening clinical status in HFpEF may not be observed or may not be as strong as in HFrEF. ${ }^{10}$ We demonstrate a strong association between 6-month survival and the overall temporal trend in NT-proBNP values in both HFpEF and HFrEF patients. Up-trending NT-proBNP values are associated with worse 6 -month survival in both subsets of HF. Conversely, downtrending NT-proBNP levels are associated with better 6-month survival rates.

\section{Author Contributions:}

Nishant Sahni MD,MS was responsible for concept, design, data analysis, and drafting the article. Rashi Arora MD: drafting, approval and critical review. Umesh Sharma MD: critical review and approval.

\section{References:}

1. Desai AS. Serial natriuretic peptide measurements are not useful in heart failure management: The art of medicine remains long.Circulation . 2013;127(4):509-516. doi:10.1161/CIRCULATIONAHA.112.120493.

2. Januzzi JL, Troughton R. Are serial BNP measurements useful in heart failure management? Serial natriuretic peptide measurements are useful in heart failure management. Circulation . 2013;127(4):500-7; discussion 508. doi:10.1161/CIRCULATIONAHA.112.120485.

3. Masson S, Latini R, Anand IS, et al. Prognostic Value of Changes in N-Terminal Pro-Brain Natriuretic Peptide in Val-HeFT (Valsartan Heart Failure Trial). J Am Coll Cardiol . 2008;52(12):997-1003. doi:10.1016/j.jacc.2008.04.069.

4. Bettencourt P, Azevedo A, Pimenta J, Friões F, Ferreira S, Ferreira A. N-terminal-pro-brain natriuretic peptide predicts outcome after hospital discharge in heart failure patients. Circulation . 2004;110(15):21682174. doi:10.1161/01.CIR.0000144310.04433.BE.

5. Anand IS, Fisher LD, Chiang YT, et al. Changes in brain natriuretic peptide and norepinephrine over time and mortality and morbidity in the Valsartan Heart Failure Trial (Val-HeFT). Circulation . 2003;107(9):12781283. doi:10.1161/01.CIR.0000054164.99881.00.

6. Jessup M, Marwick TH, Ponikowski P, Voors AA, Yancy CW. 2016 ESC and ACC/AHA/HFSA heart failure guideline update [mdash] what is new and why is it important? Nat Rev Cardiol . 2016;13(10):623-628. doi:10.1038/nrcardio.2016.134.

7. Quan H, Sundararajan V, Halfon P, et al. Coding algorithms for defining comorbidities in ICD-9-CM and ICD-10 administrative data.Med Care . 2005;43(11):1130-1139. doi:10.1097/01.mlr.0000182534.19832.83.

8. Richards AM, Januzzi Jr. JL, Troughton RW. Natriuretic peptides in heart failure with preserved ejection fraction. Hear Fail Clin . 2014;10:453-470. doi:10.1016/j.hfc.2014.04.006.

9. Maisel A, Barnard D, Jaski B, et al. Primary results of the HABIT trial (heart failure assessment with BNP in the home). $J$ Am Coll Cardiol . 2013;61(16):1726-1735. doi:10.1016/j.jacc.2013.01.052.

10. Araújo JP, Azevedo A, Lourenço P, Rocha-Gonçalves F, Ferreira A, Bettencourt P. Intraindividual variation of amino-terminal pro-B-type natriuretic peptide levels in patients with stable heart failure. $A m J$ Cardiol . 2006;98(9):1248-1250. doi:10.1016/j.amjcard.2006.06.017.

11. Dhaliwal AS, Deswal A, Pritchett A, et al. Reduction in BNP Levels With Treatment of Decompensated Heart Failure and Future Clinical Events. J Card Fail . 2009;15(4):293-299. 


\author{
Variables \\ Number of Patients \\ Black -n (\%) \\ Peripheral vascular disease $-\mathrm{n}(\%)$ \\ Dementia -n (\%) \\ Pulmonary disease $-\mathrm{n}(\%)$ \\ Diabetes Mellitus -n (\%) \\ Diabetes Mellitus with Complications-n (\%) \\ Cancer $-\mathrm{n}(\%)$ \\ Liver-Severe $-\mathrm{n}(\%)$ \\ Metastatic malignancy-n TRUE (\%) \\ $\mathrm{HIV}-\mathrm{n}(\%)$ \\ Liver disease- Mild $-\mathrm{n}(\%)$ \\ $\mathrm{CHF}-\mathrm{n}(\%)$ \\ $\mathrm{HFpEF}-\mathrm{n}(\%)$ \\ $\mathrm{HFrEF}-\mathrm{n}(\%)$ \\ Mixed $\mathrm{HF}-\mathrm{n}(\%)$ \\ Ischemic heart disease $-\mathrm{n}(\%)$ \\ Valvular heart disease $-\mathrm{n}(\%)$ \\ Cardiomyopathy $-\mathrm{n}(\%)$ \\ Maximum_NT-proBNP (median [IQR]) \\ Initial NT-proBNP (mean (sd)) \\ Maximum creatinine (median [IQR]) \\ Maximum EF (\%,mean (sd)) \\ Minimum_EF (\%,mean (sd)) \\ Charlson Comorbidity Score (mean (sd)) \\ Survival $<6$ months $=$ TRUE $(\%)$ \\ Mean Weight(lbs) (mean(sd))
}

FALSE
3538
$170(4.8)$
$1195(33.8)$
$286(8.1)$
$2496(70.5)$
$735(20.8)$
$670(18.9)$
$552(15.6)$
$107(3.0)$
$226(6.4)$
$8(0.2)$
$436(12.3)$
$3538(100.0)$
$2690(76.0)$
$0(0.0)$
$848(24.0)$
$2209(62.4)$
$2026(57.3)$
$1036(29.3)$
$4865.00[1750.00,12002.75]$
$4314.25(6605.13)$
$1.49[1.10,2.14]$
$58.57(5.76)$
$50.64(12.01)$
$4.81(2.22)$
$529(15.0)$
$189.03(60.25)$

TRUE

1665

$106(6.4) \quad 0.023$

$552(33.2) \quad 0.68$

$121(7.3) \quad 0.333$

$1033(62.0)<0.001$

$393(23.6) \quad 0.023$

$310(18.6) \quad 0.813$

$240(14.4) \quad 0.284$

$34(2.0) \quad 0.052$

$68(4.1) \quad 0.001$

$7(0.4) \quad 0.346$

$209(12.6) \quad 0.85$

$1665(100.0) \quad \mathrm{NA}$

$0(0.0) \quad<0.001$

$1665(100.0) \quad<0.001$

$0(0.0) \quad<0.001$

$1351(81.1) \quad<0.001$

$1035(62.2) \quad 0.001$

$1238(74.4) \quad<0.001$

$4760.00[1690.00,11500.00] \quad 0.39$

$7993.94(13108.46) \quad<0.001$

$1.50[1.17,2.17] \quad 0.014$

$31.20(10.14) \quad<0.001$

$25.77(10.24) \quad<0.001$

$4.47(2.05) \quad<0.001$

$262(15.7) \quad 0.488$

$188.23(55.20) \quad 0.647$

Supplementary Table 1. Demographic, clinical, biochemical and echocardiographic characteristics of patients stratified by type of CHF (HefPEF, HFrEF).

*848 patients moved between the HFpEF and HFrEF categories based on their EF in the study period were classified into a separate Mixed category. 